\title{
Synthesis of CNOT-Dihedral circuits with optimal number of two qubit gates
}

\author{
Shelly Garion ${ }^{1}$ and Andrew W. Cross ${ }^{2}$ \\ ${ }^{1}$ IBM Quantum, IBM Research Haifa, Haifa University Campus, Mount Carmel, Haifa, 3498825, Israel \\ ${ }^{2}$ IBM Quantum, IBM T.J. Watson Research Center, Yorktown Heights, NY 10598, USA
}

In this note we present explicit canonical forms for all the elements in the twoqubit CNOT-Dihedral group, with minimal numbers of controlled- $S(C S)$ and controlled- $X(C X)$ gates, using the generating set of quantum gates $[X, T, C X, C S]$. We provide an algorithm to successively construct the n-qubit CNOT-Dihedral group, asserting an optimal number of controlled- $X(C X)$ gates. These results are needed to estimate gate errors via nonClifford randomized benchmarking and may have further applications to circuit optimization over fault-tolerant gate sets.

\section{Introduction}

Randomized Benchmarking (RB) [23-25] is a well-known algorithm that provides an efficient and reliable experimental estimation of an average error-rate for a set of quantum gate operations, by running sequences of random gates from the Clifford group that should return the qubits to the initial state. RB techniques are scalable to many qubits since the Clifford group can be efficiently simulated (in polynomial time) using a classical computer $[1,10,20,27]$. RB can also be used to characterize specific interleaved gate errors [26], coherence errors [28, 31] and leakage errors [32]. RB methods were generalized to certain single qubit non-Clifford gates, like the $T$-gate [12]. In [14] the authors presented a scalable RB procedure to benchmark important non-Clifford gates, such as the controlled- $S$ gate and controlled-controlled- $Z$ gate, which belong to a certain group called the CNOT-Dihedral group.

Certain CNOT-Dihedral groups have two key

Shelly Garion: shelly@il.ibm.com

Andrew W. Cross: awcross@us.ibm.com characteristics in common with the Clifford group. First, these groups have elements with concise representations that can be efficiently manipulated [4, 14]. Second, these groups are the set of transversal (fault-tolerant) gates for certain quantum error-correcting codes $[6,7,9$, 19, 22, 34]. Since the Clifford gates together with the $T$ gate form a universal set of gates, there are many papers aiming to optimize the number of $T$ gates $[11,18,21,29,30]$. Additional methods aim to minimize the count of controlled- $X(C X)$ gates in universal circuits [33], and in particular, in controlled- $X$-phase circuits $[3,15]$.

In addition, as the Clifford gate together with the controlled- $S(C S)$ gate also forms a universal set of gates, an algorithm has recently been introduced to construct a circuit with an optimal number of $C S$ gates given a two-qubit Clifford $+C S$ operator [17]. Another example is the controlledcontrolled- $Z$ gate, which is equivalent to the Toffoli gate (up to single qubit gates), that can be decomposed into $6 C X$ gates and single qubit gates, but requires only 5 two-qubit gates in its decomposition if the $C S$ and $C S^{-1}$ gates are also available [5].

It is therefore important to efficiently present the elements in the CNOT-Dihedral group using a minimal number of physical basic gates, in particular, two-qubit gates like the controlled- $X$ $(C X)$ and controlled- $S(C S)$ gates.

Recall that $X$ is the Pauli gate defined as

$$
X=\left(\begin{array}{ll}
0 & 1 \\
1 & 0
\end{array}\right)
$$

Fix an integer $m$ and define

$$
T(m)=\left(\begin{array}{cc}
1 & 0 \\
0 & e^{2 \pi i / m}
\end{array}\right)
$$

By abuse of notation we will denote $T=T(m)$, although the $T$ gate is usually defined as $T(8)=$ 
$\left(\begin{array}{cc}1 & 0 \\ 0 & e^{2 \pi i / 8}\end{array}\right)$

The single-qubit Dihedral group is generated by the $X$ and $T=T(m)$ gates (up to a global phase) and contains $2 m$ elements,

$$
\begin{aligned}
& \langle X, T\rangle /\langle\lambda I: \lambda \in \mathbb{C}\rangle= \\
& \left\{X^{l} T^{k}: l \in\{0,1\}, k \in\{0, \ldots, m-1\}\right\} .
\end{aligned}
$$

More generally, the CNOT-Dihedral group on $n$ qubits $G=G(m)$ is generated by the gates $X$, $T=T(m)$ and controlled- $X(C X)$, up to a global phase (see [14] for details),

$$
\begin{aligned}
G=G(m)= & \left\langle X_{i}, T_{i}, C X_{i, j}:\right. \\
& i, j \in\{0, \ldots, n-1\}\rangle / \\
& \langle\lambda I: \lambda \in \mathbb{C}\rangle,
\end{aligned}
$$

where the controlled- $X(C X)$ gate is defined as

$$
C X=\left(\begin{array}{llll}
1 & 0 & 0 & 0 \\
0 & 1 & 0 & 0 \\
0 & 0 & 0 & 1 \\
0 & 0 & 1 & 0
\end{array}\right)
$$

When $m$ is not a power of 2 , the group $G=$ $G(m)$ has double exponential order as a function of the number of qubits $n$. In the special case when $m$ is a power of two, the group is only exponentially large and we can represent its elements efficiently (see [14]). Elements of $G(m)$ belong to level $\log _{2} m$ of the Clifford hierarchy when $m$ is a power of two $[19,22]$ and this is related to the fact that they are the transversal gates of certain $m$-dimensional quantum codes [7].

Again, by abuse of notation we denote $S=$ $T^{2}=T(m)^{2}=\left(\begin{array}{cc}1 & 0 \\ 0 & e^{4 \pi i / m}\end{array}\right)$, although the $S$ gate is usually defined as $T(8)^{2}=T(4)=\left(\begin{array}{ll}1 & 0 \\ 0 & i\end{array}\right)$. Observe that $S$ has order $m / 2$ if $m$ is even, and order $m$ if $m$ is odd, namely, $S$ has order $m / d$ where $d=\operatorname{gcd}(m, 2)$.

The controlled- $S(C S)$ gate belongs to $G$ and can be written as

$$
\begin{aligned}
C S_{i, j} & =T_{i} T_{j} \cdot C X_{i, j} \cdot I_{i} T_{j}^{\dagger} \cdot C X_{i, j} \\
& =\left(\begin{array}{cccc}
1 & 0 & 0 & 0 \\
0 & 1 & 0 & 0 \\
0 & 0 & 1 & 0 \\
0 & 0 & 0 & e^{4 \pi i / m}
\end{array}\right),
\end{aligned}
$$

where $T_{i} T_{j}$ means the tensor product $T_{i} \otimes T_{j}$. In the case where $m=8$, the $C S$ gate is less expensive to physically implement than one $C X$ gate $^{1}$ which makes it an alternative to $C X$ for improving circuit decompositions.

We focus on the case where $n=2$. The following two Theorems provide canonical forms for all the elements in the two-qubit CNOT-Dihedral group, such that the numbers of $C S$ and $C X$ gates are optimal. This is analogous to the description in [13] of the elements in the two-qubit Clifford group.

Theorem 1. Consider the CS-Dihedral subgroup on two qubits, namely the two-qubit group generated by the gates $X, T=T(m)$ and $C S$ (controlled-S), where $S=T^{2}$, and denote $d=$ $\operatorname{gcd}(m, 2)$. Then this group has $\frac{4 m^{3}}{d}=\frac{m}{d}(2 m)^{2}$ elements of the following form:

$$
U=C S_{0,1}^{e} \cdot X_{0}^{k} X_{1}^{k^{\prime}} \cdot T_{0}^{l} T_{1}^{l^{\prime}}
$$

where $k, k^{\prime} \in\{0,1\}, l, l^{\prime} \in\{0, \ldots, m-1\}, e \in$ $\{0,1, \ldots, m / d-1\}=\left\{0, \pm 1, \pm 2, \ldots, \pm\left\lceil\frac{m-d}{2 d}\right\rceil\right\}$.

Theorem 2. Let $G$ be the two-qubit CNOTDihedral group generated by the gates $X, T=$ $T(m), C X$ and $C S$, where $S=T^{2}$, and denote $d=\operatorname{gcd}(m, 2)$. Then this group has $24 \cdot \mathrm{m}^{3} / d$ elements, divided into the following four classes.

1. The first class is the CS-Dihedral subgroup described in Theorem 1 and has $\frac{4 m^{3}}{d}$ elements, that can be written with no $C X$ gates.

2. The second class, called the $\mathbf{C X}$-like class, consists of $\frac{8 m^{3}}{d}=2 \cdot \frac{m}{d} \cdot(2 m)^{2}$ elements, and contains all the elements of the following form, which require exactly one $C X$ gate.

$$
U=X_{0}^{k} X_{1}^{k^{\prime}} \cdot T_{0}^{l} T_{1}^{l^{\prime}} \cdot C X_{i, j} \cdot I_{i} T_{j}^{e}
$$

3. The third class, called the Double-CX-like class, consists of $\frac{8 m^{3}}{d}=2 \cdot \frac{m}{d} \cdot(2 m)^{2}$ elements, and contains all the elements of the following form, which require exactly two $C X$ gates.

$$
U=X_{0}^{k} X_{1}^{k^{\prime}} \cdot T_{0}^{l} T_{1}^{l^{\prime}} \cdot C X_{i, j} \cdot C X_{j, i} \cdot I_{i} T_{j}^{e}
$$

${ }^{1} \mathrm{Up}$ to single-qubit rotations, the gate is equivalent to controlled- $\sqrt{X}$ gate, so it can be implemented by evolving for half the duration of a controlled- $X$ gate [16]. 
4. The fourth class, called the Triple-CX-like class, consists of $\frac{4 m^{3}}{d}=\frac{m}{d} \cdot(2 m)^{2}$ elements, and contains all the elements of the following form, which require exactly three $C X$ gates.

$$
U=X_{0}^{k} X_{1}^{k^{\prime}} \cdot T_{0}^{l} T_{1}^{l^{\prime}} \cdot C X_{0,1} \cdot C X_{1,0} \cdot I_{0} T_{1}^{e} \cdot C X_{0,1}
$$

where $k, k^{\prime} \in\{0,1\}, l, l^{\prime} \in\{0, \ldots, m-1\}, e \in$ $\{0, \ldots, m / d-1\}$ and $(i, j) \in\{(0,1),(1,0)\}$.

The following Theorem provides an algorithm to successively construct the $n$-qubit CNOTDihedral group. It is analogous to [8] that discusses the generation of the $n$-qubit Clifford group. Case (1) of this Theorem shows that one can successively construct the CNOT-Dihedral group asserting an optimal number of $C X$ gates, with a bound on the space to search these group elements (see Remark 4). Moreover, one can also use the "meet in the middle" algorithm of [2] to synthesize gate sequences for the non-Clifford RB.

Theorem 3. Let $G=G(m)$ be the CNOTDihedral group on $n$ qubits, and denote $d=$ $\operatorname{gcd}(m, 2)$.

1. Let $F(r)$ be the subset of operators implementable by a circuit with $r C X$ gates (and any number of $X$ and $T$ gates). Suppose $U$ is in $F(r+1)$, then

$$
U=I_{i} T_{j}^{l} \cdot C X_{i, j} \cdot U^{\prime}
$$

for some $U^{\prime} \in F(r), i, j \in\{0, \ldots, n-1\}$, $i \neq j, l \in\{0, \ldots, m / d-1\}$. In particular,

$$
|F(r+1)| \leq \frac{m\left(n^{2}-n\right)}{d}|F(r)|
$$

2. Let $H(r)$ be the subset of operators implementable by a circuit with $r C S$ or $C S^{\dagger}$ gates (and any number of $X$ and $T$ gates). Suppose $U$ is in $H(r+1)$, then

$$
U=C S_{i, j}^{e} \cdot U^{\prime}
$$

for some $U^{\prime} \in H(r), i, j \in\{0, \ldots, n-1\}$, $i<j, e \in\{-1,1\}$. In particular,

$$
|H(r+1)| \leq\left(n^{2}-n\right)|H(r)|
$$

Remark 4. We note that the bounds in Theorem 3 are sharp and cannot generally be improved, since there is an equality in certain cases.
Indeed, assume that $n=2$. If $H(r)$ is the subset of operators implementable by a circuit with $r C S$ gates, then $H(1)=2 \cdot H(0)$ (see Theorem 1). If $F(r)$ is the subset of operators implementable by a circuit with $r C X$ gates, then $F(1)=\frac{2 m}{d} \cdot F(0)$ (see Theorem 2).

Corollary 5. In order to generate all the elements in the n-qubit CNOT-Dihedral group $G=$ $G(m)$ having at most $r C X$ gates, the algorithm generates at most

$$
(2 m)^{n} \cdot\left(\frac{m}{d}\right)^{r} \cdot\left(n^{2}-n\right)^{r}
$$

group elements.

\section{Useful identities and the proof of Theorem 3}

Consider quantum circuits on a fixed number of qubits $n$ that are products of controlled-X gates $C X$, bit-flip gates $X$, and single-qubit phase gates $T=T(m)$ satisfying $T|u\rangle:=e^{i \pi u / m}|u\rangle$. When these gates are applied to each qubit or pairs of qubits, they generate a group $G=G(m)$ of unitary operators that is an example of a CNOT-dihedral group. An element $U \in G$ acts on the standard basis as

$$
U|x\rangle=e^{p(x)}|f(x)\rangle
$$

where $p(x)=p\left(x_{1}, \ldots, x_{n}\right)$ is a polynomial called the phase polynomial and $f(x)$ is an affine reversible function. Since $x_{j} \in \mathbb{F}_{2}$, so $x_{j}^{2}=x_{j}$, the phase polynomial is

$$
p(x)=\sum_{\alpha \subseteq\{0,1\}^{n}} p_{\alpha} x^{\alpha}
$$

where $x^{\alpha}=\prod_{j \in \alpha} x_{j}$. Furthermore, the coefficients can be chosen such that $p_{\emptyset}=0$ and $p_{\alpha} \in(-2)^{|\alpha|-1} \mathbb{Z}_{2 m}$ otherwise (see [14]).

Recall the following useful identities in the Dihedral group defined in (1) generated by the $T=T(m)$ and $X$ gates (up to a global phase),

$$
\begin{gathered}
T^{\dagger}=T^{m-1} \\
X T X=T^{\dagger} \\
T X T=X \\
T X T^{\dagger}=S X
\end{gathered}
$$

We state here some useful identities in the CNOT-Dihedral group defined in (2) regarding 
the controlled- $S(C S)$ gate. According to the definition of the $C S$ gate in (3),

$$
\begin{aligned}
C S_{i, j} & =T_{i} T_{j} \cdot C X_{i, j} \cdot I_{i} T_{j}^{\dagger} \cdot C X_{i, j} \\
& =C X_{i, j} \cdot I_{i} T_{j}^{\dagger} \cdot C X_{i, j} \cdot T_{i} T_{j}
\end{aligned}
$$

We deduce that

$$
\begin{aligned}
& C S_{i, j} \cdot C X_{i, j}=T_{i} T_{j} \cdot C X_{i, j} \cdot I_{i} T_{j}^{\dagger}, \\
& C X_{i, j} \cdot C S_{i, j}=I_{i} T_{j}^{\dagger} \cdot C X_{i, j} \cdot T_{i} T_{j}
\end{aligned}
$$

Similarly,

$$
\begin{aligned}
C S_{i, j}^{\dagger} & =T_{i}^{\dagger} T_{j}^{\dagger} \cdot C X_{i, j} \cdot I_{i} T_{j} \cdot C X_{i, j} \\
& =C X_{i, j} \cdot I_{i} T_{j} \cdot C X_{i, j} \cdot T_{i}^{\dagger} T_{j}^{\dagger}
\end{aligned}
$$

We note that according to their definition, the $C S$ and $C S^{\dagger}$ gates (as well as their powers) are symmetrical, namely,

$$
\begin{aligned}
& C S_{j, i}=C S_{i, j} \\
& C S_{j, i}^{\dagger}=C S_{i, j}^{\dagger}
\end{aligned}
$$

$T$ (and all its powers) commutes with the control and target of the $C S$ gate, namely,

$$
\begin{aligned}
& I_{i} T_{j} \cdot C S_{i, j}=C S_{i, j} \cdot I_{i} T_{j}, \\
& T_{i} I_{j} \cdot C S_{i, j}=C S_{i, j} \cdot T_{i} I_{j}, \\
& T_{i} T_{j} \cdot C S_{i, j}=C S_{i, j} \cdot T_{i} T_{j}
\end{aligned}
$$

In addition, we have the following relations between the $C S$ and $X$ gates,

$$
\begin{aligned}
& X_{i} I_{j} \cdot C S_{i, j} \cdot X_{i} I_{j}=C S_{i, j}^{\dagger} \cdot I_{i} S_{j}=I_{i} S_{j} \cdot C S_{i, j}^{\dagger} \\
& I_{i} X_{j} \cdot C S_{i, j} \cdot I_{i} X_{j}=C S_{i, j}^{\dagger} \cdot S_{i} I_{j}=S_{i} I_{j} \cdot C S_{i, j}^{\dagger} \\
& X_{i} X_{j} \cdot C S_{i, j} \cdot X_{i} X_{j}=C S_{i, j} \cdot S_{i}^{\dagger} S_{j}^{\dagger}=S_{i}^{\dagger} S_{j}^{\dagger} \cdot C S_{i, j}
\end{aligned}
$$

We shall moreover use the following identities of the $C X$ gate. $T$ (and all its powers) commutes with the control of $C X$, and $X$ (and all its powers) commutes with the target of $C X$, namely,

$$
\begin{aligned}
I_{i} X_{j} \cdot C X_{i, j} & =C X_{i, j} \cdot I_{i} X_{j}, \\
T_{i} I_{j} \cdot C X_{i, j} & =C X_{i, j} \cdot T_{i} I_{j}
\end{aligned}
$$

In addition, we have the following relation between the control of $C X$ and the $X$ gate,

$$
C X_{i, j} \cdot X_{i} I_{j} \cdot C X_{i, j}=X_{i} X_{j}
$$

Recall that the $Z$ gate is defined as $Z=$ $\left(\begin{array}{cc}1 & 0 \\ 0 & -1\end{array}\right)$. Then we have the following useful relation between the $C X$ gate and the $Z$ gate,

$$
C X_{i, j} \cdot I_{i} Z_{j} \cdot C X_{i, j}=Z_{i} Z_{j}
$$

Finally, the product $C X_{i, j} \cdot C X_{j, i}$, which is in the iSWAP-like class of Clifford gates (see [13])), satisfies the following relation,

$$
I_{i} T_{j} \cdot C X_{i, j} \cdot C X_{j, i}=C X_{i, j} \cdot C X_{j, i} \cdot T_{i} I_{j}
$$

Based on the above identities we can now prove Theorem 3.

Proof of Theorem 3. 1) There exists a product of single qubit gates $V=V_{1} \ldots V_{n}, V_{k} \in\langle X, T\rangle$ such that $U=V \cdot C X_{i, j} \cdot U^{\prime}$ for some pair of qubits $i, j$. Absorb $V_{k}$ for $k \notin\{i, j\}$ into $U^{\prime}$, namely,

$$
U=X_{i}^{k} X_{j}^{k^{\prime}} \cdot T_{i}^{l} T_{j}^{l^{\prime}} \cdot C X_{i, j} \cdot U^{\prime}
$$

for some $k, k^{\prime}, l, l^{\prime}$ and $U^{\prime} \in F(r)$. Since $T_{i}^{l}$ commutes with the control of $C X_{i, j}$ by (13), we can absorb $T_{i}^{l}$ in $U^{\prime}$. Since $X_{j}^{k^{\prime}}$ commutes with the target of $C X_{i, j}$ by (13), we can also absorb $X_{j}^{k^{\prime}}$ in $U^{\prime}$. Hence,

$$
U=X_{i}^{k} T_{j}^{l} \cdot C X_{i, j} \cdot U^{\prime}
$$

for some $k, l$ and $U^{\prime} \in F(r)$.

If $k=1$ then according to (14), $X_{i} I_{j} \cdot C X_{i, j}=$ $C X_{i, j} \cdot X_{i} X_{j}$, so we can replace $U$ by

$$
I_{i} T_{j}^{l} \cdot C X_{i, j} \cdot X_{i} X_{j} \cdot U^{\prime}=I_{i} T_{j}^{l} \cdot C X_{i, j} \cdot U^{\prime \prime}
$$

where $U^{\prime \prime} \in F(r)$. We can therefore assume that $k=0$.

If $m$ is even and $l \geq m / 2$ then $T^{m / 2}=Z$, so we can rewrite $U$ as

$$
U=I_{i} T_{j}^{l} \cdot I_{i} Z_{j} \cdot C X_{i, j} \cdot U^{\prime}
$$

for some $l<m / 2$. According to (15), $I_{i} Z_{j}$. $C X_{i, j}=C X_{i, j} \cdot Z_{i} Z_{j}$, so we can replace $U$ by

$$
I_{i} T_{j}^{l} \cdot C X_{i, j} \cdot Z_{i} Z_{j} \cdot U^{\prime}=I_{i} T_{j}^{l} \cdot C X_{i, j} \cdot U^{\prime \prime}
$$

where $U^{\prime \prime} \in F(r)$. We can therefore assume that $l<m / 2$ as needed.

2) Similarly to (1) we can assume that

$$
U=X_{i}^{k} X_{j}^{k^{\prime}} \cdot T_{i}^{l} T_{j}^{l^{\prime}} \cdot C S_{i, j}^{e} \cdot U^{\prime}
$$


for some $k, k^{\prime}, l, l^{\prime}, e= \pm 1$ and $U^{\prime} \in H(r)$. Since $T$ commutes with both control and target of $C S$ by (11), we can absorb $T_{i}^{l} T_{j}^{l^{\prime}}$ in $U^{\prime}$ and so

$$
U=X_{i}^{k} X_{j}^{k^{\prime}} \cdot C S_{i, j}^{e} \cdot U^{\prime}
$$

Now, by (10) we may assume that $i<j$, and by (12) we can absorb $X_{i}^{k} X_{j}^{k^{\prime}}$ in $U^{\prime}$ and assume that $U=C S_{i, j}^{e} \cdot U^{\prime}$ for some $i<j$ and $e= \pm 1$ as needed.

\section{The canonical forms and proofs of Theorems 1 and 2}

From now on we will now assume that $G$ is the CNOT-Dihedral group on two qubits $\{0,1\}$, and describe canonical forms of the elements in $G$. This is analogous to the description in [13] of the elements in the Clifford group on two qubits.

Proof of Theorem 1. The proof follows by induction on the number $r$ of $C S$ and $C S^{\dagger}$ gates. Since $C S$ is of order $m / d$ then necessarily $r<\left\lceil\frac{m-d}{2 d}\right\rceil$.

Let $r=0$, then any $U \in H(0)$ can be written as

$$
U=X_{0}^{k} X_{1}^{k^{\prime}} \cdot T_{0}^{l} T_{1}^{l^{\prime}}
$$

where $k, k^{\prime} \in\{0,1\}, l, l^{\prime} \in\{0, \ldots, m-1\}$, since such an element belongs to the direct product of the two single-qubit Dihedral groups.

Let $r=1$, then according to Case (2) of Theorem 3 , any $U \in H(1)$ can be written as

$$
U=C S_{0,1}^{e} \cdot X_{0}^{k} X_{1}^{k^{\prime}} \cdot T_{0}^{l} T_{1}^{l^{\prime}}
$$

where $e \in\{1,-1\}, k, k^{\prime} \in\{0,1\}, \quad l, l^{\prime} \in$ $\{0, \ldots, m-1\}$.

Now assume that the Theorem holds for $H(r)$. According to Case (2) of Theorem 3 and the induction assumption, any element $U \in H(r+1)$ can be written as

$$
U=C S_{0,1}^{e} \cdot C S_{0,1}^{e^{\prime}} \cdot U^{\prime}=C S_{0,1}^{e+e^{\prime}} \cdot U^{\prime}
$$

where $U^{\prime} \in\langle T, X\rangle, e= \pm 1$ and $e^{\prime}= \pm r$, as needed.

Note that all the elements obtained in this process are distinct, since an equality $C S_{0,1}^{e} \cdot U=$ $C S_{0,1}^{e^{\prime}} \cdot U^{\prime}$ for some $e, e^{\prime} \in\{0, \ldots, m / d-1\}$ and $U, U^{\prime} \in\langle T, X\rangle$, implies that $C S_{0,1}^{e-e^{\prime}} \in\langle T, X\rangle$, so necessarily $e=e^{\prime}$ and $U=U^{\prime}$.
Lemma 6. Let $G$ be the CNOT-Dihedral group on two qubits. Then any element in $G$ which has exactly one $C S$ gate and one $C X$ gate can be rewritten as an element with no $C S$ gates and exactly one $C X$ gate.

Proof. According to Theorem 3 we may assume w.l.o.g. that such an element $U$ can be written as a product

$$
U=\left(U^{\prime} \cdot C X_{0,1} \cdot I_{0} T_{1}^{l}\right) \cdot\left(C S_{0,1}^{e} \cdot U^{\prime \prime}\right)
$$

where $U^{\prime}, U^{\prime \prime} \in\langle T, X\rangle, l \in\{0, \ldots, m / d-1\}, e \in$ $\{1,-1\}$.

Since $T$ commutes with the control and target of $C S$ by (11), we may absorb $T_{1}$ into $U^{\prime \prime}$, and so $U$ can be rewritten as

$$
\begin{aligned}
U & =U^{\prime} \cdot C X_{0,1} \cdot C S_{0,1}^{e} \cdot U^{\prime \prime} \\
& =U^{\prime} \cdot I_{0} T_{1}^{-e} \cdot C X_{0,1} \cdot T_{0}^{e} T_{1}^{e} \cdot U^{\prime \prime}
\end{aligned}
$$

for some $U^{\prime}, U^{\prime \prime}$ by (8). Therefore, $U=U^{\prime} \cdot C X_{0,1}$. $U^{\prime \prime}$ for some $U^{\prime}, U^{\prime \prime}$, as needed.

Lemma 7. Let $G$ be the CNOT-Dihedral group on two qubits. Then any element in $G$ which has exactly one $C X$ gate and no $C S$ gates can be written either as:

$$
U=X_{0}^{k} X_{1}^{k^{\prime}} \cdot T_{0}^{l} T_{1}^{l^{\prime}} \cdot C X_{0,1} \cdot I_{0} T_{1}^{l^{\prime \prime}}
$$

or:

$$
U=X_{0}^{k} X_{1}^{k^{\prime}} \cdot T_{0}^{l} T_{1}^{l^{\prime}} \cdot C X_{1,0} \cdot T_{0}^{l^{\prime \prime}} I_{1}
$$

where $k, k^{\prime} \in\{0,1\}, l, l^{\prime} \in\{0, \ldots, m-1\}$ and $l^{\prime \prime} \in\{0, \ldots, m / d-1\}$. In particular, $G$ has $\frac{8 m^{3}}{d}=$ $2 \cdot \frac{m}{d} \cdot(2 m)^{2}$ such elements.

Proof. The proof follows from Case (1) of Theorem 3.

Note that all the elements obtained in this process are indeed distinct.

First, an equality $U \cdot C X_{0,1} \cdot I_{0} T_{1}^{l}=U^{\prime}$. $C X_{0,1} \cdot I_{0} T_{1}^{l^{\prime}}$ for some $U, U^{\prime} \in\langle T, X\rangle$ and $l, l^{\prime} \in$ $\{0, \ldots, m / d-1\}$, implies that $C X_{0,1} \cdot I_{0} T_{1}^{l^{\prime}-l}$. $C X_{0,1} \in\langle T, X\rangle$, hence either $l=l^{\prime}$ and $U=U^{\prime}$; or $m$ is even and $l-l^{\prime}=m / 2$, yielding a contradiction since $l, l^{\prime}<m / 2$.

Second, an equality $U \cdot C X_{0,1} \cdot I_{0} T_{1}^{l}=U^{\prime}$. $C X_{1,0} \cdot T_{0}^{l^{\prime}} I_{1}$ for some $U, U^{\prime} \in\langle T, X\rangle$ and $l, l^{\prime} \in$ $\{0, \ldots, m / d-1\}$, implies that $C X_{0,1} \cdot T_{0}^{-l^{\prime}} T_{1}^{l}$. $C X_{1,0} \in\langle T, X\rangle$, yielding a contradiction. 
Lemma 8. Let $G$ be the CNOT-Dihedral group on two qubits. Then any element in $G$ which has exactly two $C X$ gates and no $C S$ gates can be written either as:

$$
U=X_{0}^{k} X_{1}^{k^{\prime}} \cdot T_{0}^{l} T_{1}^{l^{\prime}} \cdot C X_{0,1} \cdot C X_{1,0} \cdot I_{0} T_{1}^{l^{\prime \prime}}
$$

or:

$$
U=X_{0}^{k} X_{1}^{k^{\prime}} \cdot T_{0}^{l} T_{1}^{l^{\prime}} \cdot C X_{1,0} \cdot C X_{0,1} \cdot T_{0}^{l^{\prime \prime}} I_{1}
$$

where $k, k^{\prime} \in\{0,1\}, l, l^{\prime} \in\{0, \ldots, m-1\}$ and $l^{\prime \prime} \in\{0, \ldots, m / d-1\}$. In particular, $G$ has $\frac{8 m^{3}}{d}=$ $2 \cdot \frac{m}{d} \cdot(2 m)^{2}$ such elements.

Proof. According to Case (1) of Theorem 3 and Lemma 7 we may assume w.l.o.g. that such an element $U$ can be written as

$$
U=I_{i} T_{j}^{l} \cdot C X_{i, j} \cdot I_{0} T_{1}^{l^{\prime}} \cdot C X_{0,1} \cdot U^{\prime}
$$

where $U^{\prime} \in\langle T, X\rangle, \quad i, j \in\{0,1\}, \quad l, l^{\prime} \in$ $\{0, \ldots, m / d-1\}$. Hence, there are two options, either $(i, j)=(0,1)$ or $(1,0)$.

1) First, assume that $(i, j)=(0,1)$, then

$$
U=I_{0} T_{1}^{l} \cdot C X_{0,1} \cdot I_{0} T_{1}^{l^{\prime}} \cdot C X_{0,1} \cdot U^{\prime}
$$

If $l^{\prime}=0$ then $U \in\langle X, T\rangle$ and we are done.

Otherwise, according to (9), $C X_{0,1} \cdot I_{0} T_{1}$. $C X_{0,1}=C S_{0,1}^{\dagger} \cdot T_{0} T_{1}$, implying that

$$
\begin{aligned}
C X_{0,1} \cdot I_{0} T_{1}^{l^{\prime}} \cdot C X_{0,1} & =\left(C X_{0,1} \cdot I_{0} T_{1} \cdot C X_{0,1}\right)^{l^{\prime}} \\
& =\left(C S_{0,1}^{\dagger} \cdot T_{0} T_{1}\right)^{l^{\prime}} \\
& =C S_{0,1}^{-l^{\prime}} \cdot T_{0}^{l^{\prime}} T_{1}^{l^{\prime}}
\end{aligned}
$$

by (11). Thus we can write $U$ as an element in the subgroup generated by $C S, X$ and $T$.

Then we are done by Theorem 1 .

2) Now, assume that $(i, j)=(1,0)$, then we can write $U$ as

$$
U=T_{0}^{l} I_{1} \cdot C X_{1,0} \cdot I_{0} T_{1}^{l^{\prime}} \cdot C X_{0,1} \cdot U^{\prime}
$$

By (13), $T_{1}$ commutes with $C X_{1,0}$, so we may write $U$ as

$$
U=T_{0}^{l} T_{1}^{l^{\prime}} \cdot C X_{1,0} \cdot C X_{0,1} \cdot U^{\prime}
$$

According to (16), $T_{0} I_{1} \cdot C X_{1,0} \cdot C X_{0,1}=C X_{1,0}$. $C X_{0,1} \cdot I_{0} T_{1}$, so we can absorb $T_{0}$ in $U^{\prime}$.

Therefore,

$$
U=I_{0} T_{1}^{l^{\prime}} \cdot C X_{1,0} \cdot C X_{0,1} \cdot U^{\prime}
$$

for some $U^{\prime} \in\langle X, T\rangle$ and $l^{\prime} \in\{0, \ldots, m / d-1\}$ as needed.

Similar argument as in the proof of Lemma 7 shows that all the elements obtained in this process are indeed distinct.

First, an equality $U \cdot C X_{0,1} \cdot C X_{1,0} \cdot I_{0} T_{1}^{l}=$ $U^{\prime} \cdot C X_{0,1} \cdot C X_{1,0} \cdot I_{0} T_{1}^{l^{\prime}}$ for some $U, U^{\prime} \in\langle T, X\rangle$ and $l, l^{\prime} \in\{0, \ldots, m / d-1\}$, implies that $C X_{0,1}$. $C X_{1,0} \cdot I_{0} T_{1}^{l^{\prime}-l} \cdot C X_{1,0} \cdot C X_{0,1} \in\langle T, X\rangle$, implying that $l=l$ and $U=U^{\prime}$.

Second, an equality $U \cdot C X_{0,1} \cdot C X_{1,0} \cdot I_{0} T_{1}^{l}=$ $U^{\prime} \cdot C X_{1,0} \cdot C X_{0,1} \cdot T_{0}^{l^{\prime}} I_{1}$ for some $U, U^{\prime} \in\langle T, X\rangle$ and $l, l^{\prime} \in\{0, \ldots, m / d-1\}$, implies that $C X_{0,1}$. $C X_{1,0} \cdot T_{0}^{-l^{\prime}} T_{1}^{l} \cdot C X_{0,1} \cdot C X_{1,0} \in\langle T, X\rangle$, yielding a contradiction.

Lemma 9. Let $G$ be the CNOT-Dihedral group on two qubits. Then any element in $G$ which has exactly three $C X$ gates and no $C S$ gates can be written as:

$U=X_{0}^{k} X_{1}^{k^{\prime}} \cdot T_{0}^{l} T_{1}^{l^{\prime}} \cdot C X_{0,1} \cdot C X_{1,0} \cdot I_{0} T_{1}^{l^{\prime \prime}} \cdot C X_{0,1}$ where $k, k^{\prime} \in\{0,1\}, l, l^{\prime} \in\{0, \ldots, m-1\}$ and $l^{\prime \prime} \in\{0, \ldots, m / d-1\}$. In particular, $G$ has $\frac{4 m^{3}}{d}=$ $\frac{m}{d} \cdot(2 m)^{2}$ such elements.

Proof. According to Case (1) of Theorem 3 and Lemma 8 we may assume w.l.o.g. that such an element $U$ can be written as

$$
\begin{aligned}
& U=I_{i} T_{j}^{l} \cdot C X_{i, j} \cdot I_{0} T_{1}^{l^{\prime}} \cdot C X_{1,0} \cdot C X_{0,1} \cdot U^{\prime} \\
& \text { where } U^{\prime} \in\langle T, X\rangle, \quad i, j \in\{0,1\}, \quad l, l^{\prime} \in \\
& \{0, \ldots, m / d-1\} .
\end{aligned}
$$

Hence, there are two options, either $(i, j)=$ $(0,1)$ or $(1,0)$.

1) First, assume that $(i, j)=(1,0)$, then

$$
U=T_{0}^{l} I_{1} \cdot C X_{1,0} \cdot I_{0} T_{1}^{l^{\prime}} \cdot C X_{1,0} \cdot C X_{0,1} \cdot U^{\prime}
$$

By (13), $T_{1}$ commutes with $C X_{1,0}$, so we can write $U$ as

$$
\begin{aligned}
U & =T_{0}^{l} I_{1} \cdot I_{0} T_{1}^{l^{\prime}} \cdot C X_{1,0} \cdot C X_{1,0} \cdot C X_{0,1} \cdot U^{\prime} \\
& =T_{0}^{l} T_{1}^{l^{\prime}} \cdot C X_{0,1} \cdot U^{\prime}
\end{aligned}
$$

Then we actually have only one $C X$ gate and we are done by Lemma 7 .

2) Now assume that $(i, j)=(0,1)$, then we can write $U$ as

$$
U=I_{0} T_{1}^{l} \cdot C X_{0,1} \cdot I_{0} T_{1}^{l^{\prime}} \cdot C X_{1,0} \cdot C X_{0,1} \cdot U^{\prime}
$$


for some $U^{\prime}, U^{\prime \prime}, l, l^{\prime}$.

By (13), $T_{1}$ commutes with $C X_{1,0}$, so we can rewrite $U$ as

$$
U=I_{0} T_{1}^{l} \cdot C X_{0,1} \cdot C X_{1,0} \cdot I_{0} T_{1}^{l^{\prime}} \cdot C X_{0,1} \cdot U^{\prime}
$$

According to (16), $I_{0} T_{1} \cdot C X_{0,1} \cdot C X_{1,0}=C X_{0,1}$. $C X_{1,0} \cdot T_{0} I_{1}$, therefore,

$$
U=C X_{0,1} \cdot C X_{1,0} \cdot T_{0}^{l} T_{1}^{l^{\prime}} \cdot C X_{0,1} \cdot U^{\prime}
$$

for some $l, l^{\prime} \in\{0, \ldots, m / d-1\}$.

Now, by (13), $T_{0}$ commutes with $C X_{0,1}$ and so we can absorb $T_{0}$ in $U^{\prime}$, thus

$$
\begin{aligned}
U & =C X_{0,1} \cdot C X_{1,0} \cdot I_{0} T_{1}^{l^{\prime}} \cdot C X_{0,1} \cdot U^{\prime} \\
& =C X_{0,1} \cdot I_{0} T_{1}^{l^{\prime}} \cdot C X_{1,0} \cdot C X_{0,1} \cdot U^{\prime}
\end{aligned}
$$

by using (13) again.

The same argument as in the proof of Lemma 8 shows that all the elements obtained in this process are indeed distinct.

Proof of Theorem 2. According to Corollary 1 in [14], the CNOT-Dihedral group $G=G(m)$ on two qubits has exactly $24 \cdot \mathrm{m}^{3} / d$ elements.

By Lemma 6 , there are no elements with both $C X$ and $C S$ gates. The cases where there are only $C S$ gates were handled in Theorem 1 . The remaining cases where there are only $C X$ gates were proved in Lemmas 7, 8 and 9.

\section{References}

[1] Scott Aaronson and Daniel Gottesman. Improved simulation of stabilizer circuits. Phys. Rev. A, 70:052328, Nov 2004. DOI: 10.1103/PhysRevA.70.052328. URL https://link.aps.org/doi/10.1103/ PhysRevA.70.052328.

[2] M. Amy, D. Maslov, M. Mosca, and M. Roetteler. A meet-in-the-middle algorithm for fast synthesis of depth-optimal quantum circuits. IEEE Transactions on Computer-Aided Design of Integrated Circuits and Systems, 32(6):818-830, 2013. DOI: 10.1109/TCAD.2013.2244643.

[3] Matthew Amy, Parsiad Azimzadeh, and Michele Mosca. On the controlled-NOT complexity of controlled-NOT-phase circuits. Quantum Science and Technology, 4 (1):015002, sep 2018. DOI: 10.1088/20589565/aad8ca. URL https://doi.org/10. 1088\%2F2058-9565\%2Faad8ca.
[4] Matthew Amy, Jianxin Chen, and Neil J. Ross. A finite presentation of cnot-dihedral operators. Electronic Proceedings in Theoretical Computer Science, 266:84-97, 2018. DOI: 10.4204/eptcs.266.5. URL https://app.dimensions.ai/details/ publication/pub.1101260386andhttps: //arxiv.org/pdf/1701.00140.

[5] Adriano Barenco, Charles H. Bennett, Richard Cleve, David P. DiVincenzo, Norman Margolus, Peter Shor, Tycho Sleator, John A. Smolin, and Harald Weinfurter. Elementary gates for quantum computation. Phys. Rev. A, 52:34573467, Nov 1995. DOI: 10.1103/PhysRevA.52.3457. URL https://link.aps. org/doi/10.1103/PhysRevA.52.3457.

[6] H. Bombin and M. A. Martin-Delgado. Topological computation without braiding. Phys. Rev. Lett., 98:160502, Apr 2007. DOI: 10.1103/PhysRevLett.98.160502. URL https://link.aps.org/doi/10.1103/ PhysRevLett.98.160502.

[7] Héctor Bombín. Gauge color codes: optimal transversal gates and gauge fixing in topological stabilizer codes. New Journal of Physics, 17(8):083002, aug 2015. DOI: 10.1088/1367-2630/17/8/083002. URL https://doi.org/10.1088\%2F1367-2630\% 2F $17 \% 2 \mathrm{~F} 8 \% 2 \mathrm{~F} 083002$.

[8] Sergey Bravyi. Compiling clifford operators.

[9] Sergey Bravyi and Robert König. Classification of topologically protected gates for local stabilizer codes. Phys. Rev. Lett., 110:170503, Apr 2013. DOI: 10.1103/PhysRevLett.110.170503. URL https://link.aps.org/doi/10.1103/ PhysRevLett.110.170503.

[10] Sergey Bravyi and Dmitri Maslov. Hadamard-free circuits expose the structure of the clifford group, 2020. URL https://arxiv.org/abs/2003.09412.

[11] Earl T. Campbell and Mark Howard. Unifying gate synthesis and magic state distillation. Phys. Rev. Lett., 118:060501, Feb 2017. DOI: $\quad$ 10.1103/PhysRevLett.118.060501. URL https://link.aps.org/doi/10. 1103/PhysRevLett.118.060501.

[12] Arnaud Carignan-Dugas, Joel J. Wallman, and Joseph Emerson. Characterizing universal gate sets via dihe- 
dral benchmarking. Phys. Rev. A, 92: 060302, Dec 2015. DOI: 10.1103/PhysRevA.92.060302. URL https://link.aps . org/doi/10.1103/PhysRevA.92.060302.

[13] A. D. Córcoles, Jay M. Gambetta, Jerry M. Chow, John A. Smolin, Matthew Ware, Joel Strand, B. L. T. Plourde, and M. Steffen. Process verification of two-qubit quantum gates by randomized benchmarking. Phys. Rev. A, 87: 030301, Mar 2013. DOI: 10.1103/PhysRevA.87.030301. URL https://link.aps. org/doi/10.1103/PhysRevA.87.030301.

[14] Andrew W Cross, Easwar Magesan, Lev S Bishop, John A Smolin, and Jay M Gambetta. Scalable randomised benchmarking of non-clifford gates. npj Quantum Information, 2(1), 2016. DOI: 10.1038/npjqi.2016.12. URL https://doi. org/10.1038/npjqi.2016.12.

[15] Meuli G., Soeken M., and De Micheli G. Satbased $\{\mathrm{CNOT}, \mathrm{T}\}$ quantum circuit synthesis. Kari J., Ulidowski I. (eds) Reversible Computation. RC 2018. Lecture Notes in Computer Science, 11106, 2018. DOI: 10.1007/978-3-319-99498-7¹2.

[16] Shelly Garion, Naoki Kanazawa, Haggai Landa, David C. McKay, Sarah Sheldon, Andrew W. Cross, and Christopher J. Wood. Experimental implementation of non-clifford interleaved randomized benchmarking with a controlled-s gate, 2020. URL https://arxiv.org/abs/2007.08532.

[17] Andrew N. Glaudell, Neil J. Ross, and Jacob M. Taylor. Optimal two-qubit circuits for universal fault-tolerant quantum computation, 2020. URL https://arxiv.org/ abs/2001. 05997.

[18] David Gosset, Vadym Kliuchnikov, Michele Mosca, and Vincent Russo. An algorithm for the t-count. Quantum Info. Comput., 14(15-16):1261-1276, November 2014. ISSN 1533-7146. URL https://dl.acm. org/doi/10.5555/2685179.2685180.

[19] Daniel Gottesman and Isaac L. Chuang. Demonstrating the viability of universal quantum computation using teleportation and single-qubit operations. Nature, 402: 390-393, 1999. ISSN 1476-4687. DOI: 10.1038/46503. URL https://doi.org/ $10.1038 / 46503$.
[20] Daniel Eric Gottesman. Stabilizer codes and quantum error correction, 1997. URL https://resolver.caltech.edu/ CaltechETD : etd-07162004-113028.

[21] Luke E Heyfron and Earl T Campbell. An efficient quantum compiler that reduces $\mathrm{t}$ count. Quantum Science and Technology, 4 (1):015004, sep 2018. DOI: 10.1088/20589565/aad604. URL https://doi.org/10. 1088\%2F 2058-9565\%2Faad604.

[22] Tomas Jochym-O'Connor, Aleksander Kubica, and Theodore J. Yoder. Disjointness of stabilizer codes and limitations on fault-tolerant logical gates. Phys. Rev. X, 8:021047, May 2018. DOI: 10.1103/PhysRevX.8.021047. URL https://link.aps . org/doi/10.1103/PhysRevX.8.021047.

[23] E. Knill, D. Leibfried, R. Reichle, J. Britton, R. B. Blakestad, J. D. Jost, C. Langer, R. Ozeri, S. Seidelin, and D. J. Wineland. Randomized benchmarking of quantum gates. Phys. Rev. A, 77:012307, Jan 2008. DOI: 10.1103/PhysRevA.77.012307. URL https://link.aps.org/doi/10.1103/ PhysRevA.77.012307.

[24] Easwar Magesan, J. M. Gambetta, and Joseph Emerson. Scalable and robust randomized benchmarking of quantum processes. Phys. Rev. Lett., 106:180504, May 2011. DOI: 10.1103/PhysRevLett.106.180504. URL https://link.aps.org/doi/10.1103/ PhysRevLett.106.180504.

[25] Easwar Magesan, Jay M. Gambetta, and Joseph Emerson. Characterizing quantum gates via randomized benchmarking. Phys. Rev. A, 85:042311, Apr 2012. DOI: 10.1103/PhysRevA.85.042311. URL https://link.aps.org/doi/10.1103/ PhysRevA. 85.042311.

[26] Easwar Magesan, Jay M. Gambetta, B. R. Johnson, Colm A. Ryan, Jerry M. Chow, Seth T. Merkel, Marcus P. da Silva, George A. Keefe, Mary B. Rothwell, Thomas A. Ohki, Mark B. Ketchen, and M. Steffen. Efficient measurement of quantum gate error by interleaved randomized benchmarking. Phys. Rev. Lett., 109:080505, Aug 2012. DOI: 10.1103/PhysRevLett.109.080505. URL 
https://link.aps.org/doi/10.1103/

PhysRevLett.109.080505.

[27] D. Maslov and M. Roetteler. Shorter stabilizer circuits via bruhat decomposition and quantum circuit transformations. IEEE Transactions on Information Theory, 64(7):4729-4738, 2018. DOI: 10.1109/TIT.2018.2825602.

[28] David C. McKay, Stefan Filipp, Antonio Mezzacapo, Easwar Magesan, Jerry M. Chow, and Jay M. Gambetta. Universal gate for fixed-frequency qubits via a tunable bus. Phys. Rev. Applied, 6:064007, Dec 2016. DOI: $\quad$ 10.1103/PhysRevApplied.6.064007. URL https://link.aps.org/doi/10. 1103/PhysRevApplied.6.064007.

[29] Yunseong Nam, Neil J. Ross, Yuan Su, Andrew M. Childs, and Dmitri Maslov. Automated optimization of large quantum circuits with continuous parameters. $4: 23,2018$. ISSN 2056-6387. DOI: 10.1038/s41534-018-0072-4. URL https:// doi.org/10.1038/s41534-018-0072-4.

[30] Neil J. Ross and Peter Selinger. Optimal ancilla-free clifford $+\mathrm{t}$ approximation of z-rotations. Quantum Info. Comput., 16(11-12):901-953, September 2016. ISSN 1533-7146. URL https://dl.acm. org/doi/abs/10.5555/3179330.3179331.

[31] Joel Wallman, Chris Granade, Robin Harper, and Steven T Flammia. Estimating the coherence of noise. New Journal of Physics, 17(11):113020, nov 2015. DOI: 10.1088/1367-2630/17/11/113020. URL https ://doi .org/10.1088\%2F1367-2630\% $2 \mathrm{~F} 17 \% 2 \mathrm{~F} 11 \% 2 \mathrm{~F} 113020$.

[32] Christopher J. Wood and Jay M. Gambetta. Quantification and characterization of leakage errors. Phys. Rev. A, 97: 032306, Mar 2018. DOI: 10.1103/PhysRevA.97.032306. URL https://link.aps. org/doi/10.1103/PhysRevA.97.032306.

[33] Ed Younis, Koushik Sen, Katherine Yelick, and Costin Iancu. Qfast: Quantum synthesis using a hierarchical continuous circuit space, 2020. URL https : //arxiv . org/ $\mathrm{abs} / 2003.04462$.

[34] B. Zeng, A. Cross, and I. L. Chuang. Transversality versus universality for additive quantum codes. IEEE Transactions on
Information Theory, 57(9):6272-6284, 2011. DOI: 10.1109/TIT.2011.2161917. 